\title{
LOS PATRONES ESTETICOS \\ EN LOS ALBORES DEL SIGLO XXI \\ Hacia una revisión de los estudios en torno \\ a este tema
}

\section{Carmen Bañuelos*}

Universidad Complutense

\section{INTRODUCCION}

\section{Cuerpo y esquema corporal}

Los eruditos de la psicología corporal definen «nuestro cuerpo como algo objetivo, concreto, que se puede medir con límites precisos; pero lo que llamamos "esquema corporal" es la idea que tenemos del mismo, lo que, por el contrario, es algo subjetivo, sujeto a posibles modificaciones, a pesar de que el cuerpo no varíe entre sí».

La corporalidad se constituye en un instrumento de expresión de nuestra propia personalidad, también nos sirve para tomar contacto con el exterior, comparándonos con otros cuerpos y objetos; por lo que se puede hablar, dentro del esquema corporal, de «cuerpo-objeto», es decir, de la representación aislada que nos hacemos de nuestro cuerpo en sí mismo, y de "cuerpo-vivido", que se referiría a la forma en que nuestra corporalidad se manifiesta en nuestras relaciones humanas y la socialización.

* Este artículo está dedicado «In Memoriam»a Francisco Grande Covián, Catedrático emérito de Nutrición (recientemente fallecido), con el recuerdo más entrañable de los momentos compartidos debatiendo sobre la nutrición humana y la obsesión por adelgazar.

\section{Reis}


El concepto que tenemos de nuestro cuerpo, el esquema corporal, se elabora dependiendo de numerosos factores, que influyen modificándolo, pudiendo, en algunos casos, originar un trastorno psicopatológico del esquema corporal. Dentro de estos factores se encuentra la observación visual de nuestro cuerpo, directamente a través del espejo, junto con la información que nos llega a través del propio sistema nervioso. También influyen los sentimientos y nuestro estado de ánimo a la hora de configurar el esquema corporal, siendo la norma que nos encontremos con peor aspecto físico cuando estamos decaídos. Las ideas o pensamientos en un momento dado ("Cogniciones») ejercen tanta influencia sobre nuestro esquema corporal como los sentimientos y la vida afectiva; también el ambiente y factores de tipo sociocultural, como la moda, influyen en la idea que guardamos de nuestra corporalidad: si la moda, tal como ocurre en la actualidad, exige estar más bien delgado, podemos llegar a pensar que estamos demasiado gordos, mientras que si la moda fuese opuesta, creeríamos que estamos delgados ${ }^{1}$. Algo similar puede decirse de la información que recibimos mediante las opiniones de las personas que nos rodean. Si alguien nos dice que tenemos un supuesto defecto, especialmente si es en forma de burla, como es frecuente durante la infancia y la adolescencia, podemos establecer una gran modificación en nuestro esquema corporal que, además, puede constituir un gran trauma psíquico que origine sentimientos de inferioridad e incluso un auténtico complejo ${ }^{2}$.

\section{Trastornos en la imagen corporal: anorexia y bulimia}

No hace más de diez años, sólo en ciertos ámbitos de superespecialistas o en algunas reuniones muy minoritarias, podía oírse hablar de anorexia nerviosa. Con mayor rareza todavía, de bulimia nerviosa. Los ahora conocidos como trastornos del comportamiento alimentario parecían no existir. Pero ¿̇realmente existían? Ni la prensa hablaba de ellos, ni los medios iconográficos se recreaban con imágenes más o menos sensacionalistas de anoréxicas, ni se manifestaba estado de opinión alguno referido a estos temas. Pero el estudio de los trastornos alimentarios ha experimentado una rápida evolución en un breve espacio de tiempo. En tan sólo una década se ha pasado del esbozo en las descripciones de los síndromes, al planteamiento de teorías cada vez más sólidas. Este avance en el conocimiento ha sido promovido por la urgencia de una sociedad que demanda más medios para tratar unas alteraciones que se han constituido en un grave problema sanitario, social y estético. Mas es cierto que algo está cambiando. Ya son

1 AA.VV. (1991), El esquema corporal. Guia práctica de psicología, Madrid: Ed. Temas de Hoy, (3. ${ }^{\text {e }}$ d.), pp. 104-105.

2 S. A. Richarson, N. Goodman, A. H. Hastorf y S. M. Dornbush, "Cultural uniformity in reaction to physical disabilities», American Sociological Review, 26 (1961): 241-247. S. F. StARger y J. P. BURKe, "A reexamination of body build stereotypes», Journal of Research in Personality, 16 (1982): 435-446. 
dos las revistas internacionales dedicadas al estudio del comportamiento alimentario: International Journal of Eating Disorders y European Eating Disorders. Anualmente no bajan de cinco los congresos y reuniones científicas en todo el mundo cuyo contenido monográfico está constituido por tales trastornos.

Los clínicos y los investigadores han trabajado arduamente para encontrar aquella causa fundamental de los trastornos. Se han originado multitud de teorías más o menos contrastadas. Algunas de ellas han adquirido mucha popularidad y son de dominio público; por ejemplo, en la anorexia nerviosa fácilmente se oyen comentarios acerca de la culpabilidad del entorno familiar o la del miedo a crecer y convertirse en adulta, pero no por ser conocidas son del todo ciertas. Actualmente, nadie cree que existe una sola causa para explicar la eclosión de los trastornos alimentarios. La opinión más generalizada es que son de origen multicausal, es decir, que se ha de dar una conjunción de factores para que se pueda presentar el trastorno. Pero aún hay más, una gran mayoría de investigadores nos lo describen como un continuum en el que en un extremo estarían las personas que tienen preocupaciones por su figura y hacen dieta, pero no presentan trastornos, y en el otro extremo se hallarían las personas que presentan bien claramente establecidas la anorexia y/o bulimia u otras alteraciones. Esta última teoría está avalada por diferentes estudios y explica el gran aumento de la prevalencia de estos trastornos en las sociedades occidentales ${ }^{3}$.

La formulación de objetivos tales como "Quiero ser una Top Model» o "Quiero triunfar en la vida» viene marcada por la presión social a la delgadez. Por ejemplo, si la prevalencia de estos trastornos fuese la misma en cualquier sociedad, como sucede con otras alteraciones, podríamos pensar que se debe a causas de tipo general y que no están especialmente ligados a factores de tipo cultural, pero los numerosos estudios que se han llevado a cabo demuestran más bien lo contrario ${ }^{4}$. En las sociedades en las que se enfatiza la delgadez como primer criterio de belleza y la preocupación por la figura y el peso aparecen los trastornos alimentarios en una proporción mucho mayor.

3 Rosa M. RaICH (1994), Anorexia y bulimia: trastornos alimentarios, Madrid: Ediciones Pirámide.

${ }^{4}$ Los trastornos alimentarios es bastante raro que se den en la población negra de Africa (Famuyiwa, 1988) y la población china que vive en el Lejano Oriente (Lee y cols., 1988). Pero cuando se analiza la prevalencia de estos trastornos en la población originaria de Asia o Africa que ha emigrado y se ha establecido en el mundo occidental, aumenta dramáticamente. Ficher y cols. (1983) identifican un 1,1 por 100 de anorexia nerviosa en chicas griegas que viven en Munich, frente al 0,42 por 100 que se observa entre las que viven en Grecia. Comparando unos grupos similares en número de muchachas árabes que están estudiando, unas en El Cairo y otras en Londres, Nasser (1986) identifica seis casos de bulimia en Inglaterra, frente a ninguno en El Cairo. O. O. FamuYiwa, "Anorexia in two Nigerians», Acta Psychiatrica Escandinavica, 78 (1988): 550-554; S. LeE, H. F. K. ChIU y C. CHEN, «Anorexia nervosa in Hong Kong: Why not more in Chinese?», British Journal of Psychiatry, 154 (1989): 683-688; M. M. FICHER, «Bulimia Nervosa: Basic Research", Diagnosis and Therapy, New York: Wiley, 1990; M. NASSER, "Comparative study of the prevalence of abnormal eating attitudes among arab female students at both London and Cairo universities", Psychological Medicine, 16 (1986): 621-625. 
En la sociedad occidental, a pesar de producirse, paradójicamente, un aumento de peso, hay una marcada preferencia por la esbeltez que parece que predomina en las clases socioeconómicas altas, a diferencia de los países que tienen dificultades en la obtención del alimento. Esta presión por el ideal estético se ha ido intensificando5.

Los trastornos alimentarios no son un problema nuevo, lo novedoso es la virulencia con la que se han presentado en la sociedad actual y el cambio de actitudes que ésta ha tenido en sus conceptos de salud y de ideal estético. La anorexia y la bulimia poseen un mismo denominador común: la alteración del comportamiento alimentario. Con respecto a la bulimia podemos afirmar que es un síntoma caracterizado por episodios de atracones de comida seguidos de vómitos provocados o la toma de purgantes, y ha ido convirtiéndose desde la década de los cuarenta en un cuadro clínico con identidad propia. La emergencia de la bulimia como síndrome (bulimia nerviosa) reveló a su vez la inoperatividad de los sistemas de clasificación. Si hasta entonces se podía establecer una clara distinción entre los trastornos alimentarios en función del peso y del patrón alimentario, estos parámetros dejaron de funcionar en el caso de la bulimia nerviosa, ya que este trastorno se da en todas las categorías de peso y además se alternan los períodos de ayuno y atracones.

Hoy en día se consolidan cada vez más las dietas alimenticias, en cuyo «ascetismo», más que el de la higiene, encontramos un impulso agresivo con respecto al cuerpo. Las sociedades antiguas seguían prácticas rituales de ayuno, cuya función era la de expurgar y reabsorber a través de la observación colectiva todo el impulso agresivo que se desarrolla hacia el cuerpo. Nuestra sociedad de consumo excluye por principio toda norma restrictiva por ser incompatible con la «liberación» del cuerpo, de forma que todo «impulso agresivo antagonista que libera... y que ya no está canalizado por las instituciones sociales recae hoy en la más profunda preocupación por el físico». Es precisamente este

5 Garner y cols. (1980) revisaron el peso y la altura de las modelos de Playboy y de las concursantes y ganadoras del concurso de Miss América desde el año 1959 hasta 1978 y no sólo encontraron una disminución de peso de aproximadamente $3,4 \mathrm{Kg}$., sino una disminución en las medidas de pecho y caderas. Al mismo tiempo que los modelos de estética corporal femenina disminuían su peso, la mujer americana aumentaba en una medida de 2,8 $\mathrm{Kg}$. También estudiaron el impacto publicitario de los productos para hacer dieta y observaron su incremento. Wiseman y cols. (1992) han replicado este estudio y han deducido que los pesos de las misses no sólo han disminuido (entre 1979 y 1988), sino que están entre un 13 y un 19 por 100 por debajo del peso normativo. Toro y cols. (1989) hicieron un análisis de la publicidad "pro esbeltez» incluida en las 10 revistas «femeninas» con más de 100.000 ejemplares editados que se publican en España y observaron que casi uno de cada cuatro anuncios invitaba directa o indirectamente a alcanzar un peso más bajo. D. M. Garner, P. E. Garfinkel, D. Schwartz y M. Thompson, «Cultural expectations of thinness in women", Psychological Medicine, 10 (1980): 647-656; C. V. Wiseman, J. Gray, J. E. Mosiman y A. H. Ahrens, "Cultural expectations of thinness in women: An update», International Journal of Eating Disorders, 11 (1992): 85-89; J. TORO, M. Cervera y P. PÉrez, "Body shape, publicity \& anorexia nervosa", Social Psychiatry and Psychiatric Epidemiology, 23 (1989): 132-136. 
impulso el que, más allá de las determinaciones de la moda, alimenta esta perseverancia autodestructiva, insoportable e irracional en la cual la belleza y la elegancia, que eran las causas originales, no son nada más que una excusa para un obsesivo ejercicio cotidiano de disciplina. El cuerpo, en una total inversión de los términos, se convierte en ese objeto amenazador que hay que despertar, llevar a sus medidas justas y mortificar con fines "estéticos», con los ojos fijos en las modelos esqueléticas y demacradas de cualquier revista de moda, en las que se puede descifrar toda la contradictoria agresividad de una sociedad de la abundancia contra el triunfalismo del propio cuerpo y todas las vehementes negaciones de los propios principios.

\section{El ideal estético}

Todas las sociedades han desarrollado unos ideales de belleza que marcan los objetivos a conseguir. Dentro de estas exigencias se ha insistido especialmente en el ideal de belleza femenina ${ }^{6}$. La delimitación del modelo estético tiene un refinamiento que no podemos obviar; probablemente hoy en día creeríamos que Marilyn Monroe estaba un poco gorda. Nasser apuntaba que «estar delgada» simboliza a la mujer nueva que es capaz de combinar cualidades de autocontrol y de libertad sexual con los valores tradicionales de atractivo?

Como argumenta Mariano Alemany en su libro Obesidad y nutrición, «la influencia de los medios de comunicación en el desarrollo de los ideales estéticos es clara aunque engañosa para la inmensa mayoría de la población. Los ideales cinematográficos han cedido el paso en buena medida a otras/os modelos salidos del campo de la publicidad como Claudia Schiffer, abandonándose en parte "estilo" y actitudes o manierismos personales, el "carácter" de un personaje como el de Humphrey Bogart en Casablanca, prototipo del duro-blando heroico que ha llenado la imaginación de varias generaciones de la juventud y de los adultos de ambos sexos. Se han sustituido estos modelos por un esteticismo basado exclusivamente en la apariencia física. Hombres jóvenes, viriles, fuertes, instantáneos, conquistadores, bronceados, musculosos, sin un gramo de grasa extra, sin un pelo fuera de sitio, desodorizados, perfumados, vestidos con un peculiar cuidado desenfadados y rubios aparecen junto a mujeres jóve-

${ }^{6}$ Hay numerosos ejemplos de alteración de patrones biológicos en función de normativas sociales; sólo es necesario recordar la deformación de los pies en las chinas, el artificial alargamiento del cuello en las mujeres Karen de Burma, el ensanchamiento exagerado de los labios en la cultura ugandesa, el uso de tatuajes, etc. En nuestra cultura, hay que recordar los "miriñaques» que exaltaban un "detrás» femenino aumentado a fin de resaltar las entonces en boga "cinturas de avispa», las fajas y corsés que moldeaban las formas a la moda, disminuyendo la cintura y el vientre, hasta la puesta en escena de las piernas femeninas, anteriormente guardadas celosamente bajo las largas faldas. Los tacones altos, la falda estrecha, los tejanos ajustados no son un modelo de comodidad, pero, por conocidos, nos parecen más normales que los ejemplos anteriores.

7 M. NASSER, "Eating disorders: The cultural dimension", Social Psychiatry and Psychiatric Epidemiology, 23 (1988): 184-187. 
nes, atractivas, altas, delgadas, perfectamente peinadas, maquilladas, también desodorizadas, sin grasas y aún más perfumadas, seductoras, distantes pero asequibles, instantáneamente conquistables, bronceadas, magníficamente vestidas y generalmente rubias. A lo mejor, el "número" que realiza sirve para indicar que un coche es veloz o que el perfume que anuncia es capaz de rendir perdidamente a cualquiera a los pies de quien lo lleva. El constante bombardeo de estas imágenes llega a crear problemas de identidad y de aceptación de la propia imagen. Las personas corrientes no están demasiado lejos de los ideales estéticos de otras épocas, pero el constante mostrar otros tipos humanos - ejemplares perfectos - rodeados de todas las comodidades y ventajas imaginables, incluido un impresionante éxito sexual inmediato, hace que la confianza en uno/a mismo/a y sus capacidades sea menor. En esta unión de belleza y represión en el culto de la línea vemos una de las paradojas de nuestra civilización. El cuerpo en su materialidad y sexualidad, ya sólo tiene una función de soporte de dos leyes totalmente distintas a las de la satisfacción: el imperativo de la moda y el imperativo de la muerte. La mística de la línea, el atractivo de la esbeltez, se han enraizado profundamente en nuestra cultura precisamente como forma de violencia».

Continúa comentando Alemany en su libro que «el negocio del adelgazamiento tiene dos lecturas importantes y plenamente complementarias; por un lado, los medios de comunicación que difunden constantemente la publicidad nos abruman y agreden con ideales estéticos que son inalcanzables para la inmensa mayoría de la población. Esta acción comercial se complementa con la divulgación constante de temas sanitarios, que se caracterizan por un escaso control científico de sus contenidos. Los publicistas tienen la excusa que, con la exhibición de ejemplares humanos magníficos y perfectos, venden muchos productos y, además, crean un mercado importante de materiales para la belleza y el cuidado del cuerpo. Por el lado sanitario, se agobia al consumidor con los peligros de los aditivos, el colesterol, las grasas insaturadas, la sal, el azúcar, y tantos enemigos públicos denunciados. En muchos de los casos hay una buena parte de razón en lo que se manifiesta, pero las exageraciones y las suposiciones sin base, además de los constantes rumores mal difundidos por los medios de comunicación, hacen que el mensaje real se diluya, y adquiera dimensiones tremendistas. Si todos las/los consumidoras/res llegaran a creer lo que las personas — supuestamente autorizadas- difunden a través de los medios de comunicación, nadie comería nada. De hecho, esto ha creado temores generalizados hacia algunos de los componentes de nuestra dieta y que caen en miedos patológicos, que serían ignorados si no fuera porque a la larga pueden generar un déficit de nutrientes que sí sería peligroso. La información que podría ser considerada muy adecuada para una gran parte de la población se convierte, por la publicidad indiscriminada y exagerada, en algo aplicable a toda la población en su conjunto, siguiendo en muchos casos modas o costumbres poco serias. Sin embargo, no todos los obesos están igualmente estigmatizados. El cuerpo ha sido tradicionalmente un «valor» mucho más importante 
para la mujer que para el hombre. Por ello, las mujeres obesas están más discriminadas socialmente y presionadas por hacer dieta que los varones obesos y son ellas las que llenan las clínicas de control de peso» ${ }^{8}$.

Perpiña afirma en su libro Transtornos alimentarios que «muchos de los síntomas que caracterizan a los obesos, tales como ansiedad, depresión, pobre imagen y baja autoestima, pueden ser el resultado más que la causa de su condición obesa. Es cierto que aunque existan grupos de obesos que por causas concretas como la adolescencia, o las máximas presiones por conseguir un cuerpo delgado, sufren más desórdenes psicológicos, lo cierto es que no se ha encontrado ningún "perfil anormal de personalidad obesa", es decir, no están más perturbados psicológicamente que los sujetos con un peso normal, y no sufren una mayor incidencia de desórdenes emocionales que los no obesos. En ningún caso se puede afirmar que los rasgos de personalidad causen obesidad. Donde sí tiene sentido hablar de una posible "perturbación" psicológica de la obesidad es en las consecuencias que la obesidad tiene para el individuo. La presión del ideal estético, los estereotipos culturales están perjudicando a posteriori a gentes que no pueden desenvolverse entre los demás llevando encima un "cuerpo no grato"»?

\section{HACIA UNA SOCIOLOGIA DE LA GORDURA. UNA REVISION DE LOS ESTUDIOS EN TORNO AL TEMA}

\section{Introducción}

La gordura se puede identificar como una condición socialmente estigmatizada. Este poder se adecua con los intereses tradicionales de los sociólogos en la estratificación social, la construcción del estigma, la medicalización de la desviación, y así sucesivamente.

De hecho, en las teorías generales en estos campos frecuentemente se usa «obesidad» como un ejemplo ilustrativo que se puede comprobar en Goffman, Conrad y Schneider ${ }^{10}$. Peculiarmente, siempre ha existido un mínimo interés entre los sociólogos en estudiar la gordura. Además, muchos de los trabajos de los sociólogos/as americanos/as se han desarrollado en esta área, y han tendido a tratar la obesidad como un problema personal de la gordura en sí mismo. En general, los sociólogos/as han asumido «la verdad» de este mito popular acerca de la gordura, incluyendo nociones como: que la gordura es inevitablemente un serio peligro para la salud, que la gente está gorda porque se sobrealimentan o porque son infelices o psicológicamente anormales. Existe algún estudio socio-

Mariano Alemany (1992), Obesidad y Nutrición, Madrid: Alianza Editorial, pp. 45 y 52.

9 C. Perpiñá (1989), Trastornos alimentarios: el estado de la cuestión, Valencia: Promolibro.

${ }^{10}$ Goffman (1963), Estigma, Anagrama, 1980. Conrad y SCHneIder, Desviance and medicalization, Mosby, 1980. 
lógico que tiene que ver con actitudes hacia la gente gorda, y un pequeño número de investigadores con trazos de carrera moral hacia las personas gordas. La sociología cotidiana, a veces, en su mayor parte, ignora la cuestión de la construcción social de la gordura como un problema; las/os estudiosas/os que coinciden con esta cuestión, fundamentalmente sociológica, han sido feministas, gordos/as liberacionistas, psicólogos, retóricos, filosóficos e historiadores.

Realizaremos un acercamiento crítico a la sociología de la gordura. Lo ligero de su acceso sería una revisión previa de las investigaciones de la gordura por sociólogos/as, incluyendo el bienestar social, la epidemiología social y la estigmatización. Revisaremos el trabajo relevante desde otras disciplinas, especialmente los estudios feministas y la historia cultural.

\section{Un acceso critico al estudio de la Sociología de la Gordura}

En la introducción de La cultura como problema público, Joseph Gusfield discute el concepto de posesión de los problemas públicos:

«El concepto de posesión de los problemas públicos deriva del reconocimiento que en las áreas de opinión pública y debate de todos los grupos no tienen igual poder, influencia y autoridad que define la realidad del problema. La habilidad de crear y la influencia de la definición pública de un problema es que yo infiero esto como una posesión ${ }^{11}$.

Claramente, en los problemas públicos que genera la gordura en la América contemporánea, la medicina es la gran titular y poseedora de existencias. Esto está reflejado por encima, en los términos que se usan para prácticamente todo, en la discusión de problemas: mientras el uso común de la terminología en torno a la gordura, en el pasado reciente, ha preferido los términos «regordete», "grande», o «rechoncho», "grandote» y «obeso», ahora están en ascenso. Muchos escritores acerca de la gordura —incluidos los científicos socialesusan estos últimos términos, e igualmente usan las ideas tal y como las manifiestan los médicos, como por ejemplo que la gordura es un problema médico cuya única salida es la pérdida de peso.

Todavía la tradición crítica de la sociología ha constatado que los problemas sociales tienen historia, que el entender la vida social significa que nosotros debemos examinar lo que está reconocido por los hechos sociales.

La idea de que la gordura es un problema médico es justo un hecho de asunción que requiere un cuestionamiento crítico. Los sociólogos/as deberían ver que nuestra tarea no soluciona los problemas de obesidad, pero la exploración de cómo hacerse gordo/a, y haciéndolo, definiríamos la obesidad.

${ }^{11}$ GUSFIELD, The culture of public problems: drinking-driving and the symbolic order, University of Chicago Press, 1981, p. 10. 
Existe otra terminología popular que se usa en el ámbito de la gordura: el término "gordo" por él mismo. La extensión de la estigmatización de la gordura en esta cultura es relevante en parte por "gorda", la cual no es usual por una persona atenta que describe a otra, y nunca se usa en la literatura académica. La liberación de los gordos/as, a menudo, ha tendido a reclamar esta palabra, argumentando que el problema no está sustentado en la condición descrita, pero sí en las actitudes que describen. Ellos compiten con la medicina, y con muchas fuerzas difusas de estéticos y moralistas, pero el poder define la realidad. Claramente aquí están reflejados los problemas críticos por sociólogos: ¿Por qué es peyorativo "gordo/a", por cómo es de peyorativo o cómo empezó siéndolo? ¿Cómo son los efectos sociales que definen al gordo/a como feo/a, versus definido como pecado, versus definido como enfermedad? Lo delicado de este acceso crítico a la sociología de la gordura sería el desacuerdo en el marco de las investigaciones sociológicas fechadas en el sujeto. El tópico ha sido sorprendentemente poco estudiado, y también como lo han considerado muchos de los estudiosos/as, que ha sido asumiéndolo de la manera más común. Todavía hay brillantes excepciones, y muchos alentadores trabajos hechos por estudiosos/as en otras disciplinas. Nos permitiremos empezar a examinar la literatura más relevante al detalle.

\section{El acceso al trabajo social}

La mayoría de los artículos de prensa concernientes a la gordura tratan el acceso al trabajo social, esto es, hacen referencia a sus problemas, efectivamente refiriéndose a eliminar la obesidad ${ }^{12}$. Este tipo de investigaciones tienen un pequeño interés sociológico, desde éstos no se dirige la cuestión social de la gordura. Una excepción interesante es Joan Rockwell, multicampo de estudio de los weight watchers. A pesar de la pérdida de peso de la orientación de las investigaciones, Rockwell echa un vistazo a estas cuestiones acerca de la visión del enojo cultural de la gordura. Ella informa que los weight watchers (desde 1976) hacen programas en los que ellos llaman la «rica industria mundial»: Reino Unido, Europa Occidental, y áreas asociadas de hecho con Australia, Nueva Zelanda, Japón, Sudáfrica y Rhodesia, y miembros abrumadoramente blancos, clases medias y mujeres ${ }^{13}$.

Una parte significativa de los estudios están interesados en la epidemiología social de la gordura. Otra vez, en estos estudios, obesidad está implícita y explícitamente definida como un problema de salud, y la asunción fundamen-

12 Hamilton y Zimmerman, "The interaction of marital power and weight and self esteem with courtship behaviour», Journal of Social Service Research, vol. 8 (1985): 51-64. STEVENSON, "Obesity treatment: research and application», Social Work in Health Care, 4 (1978): 165-178; ZAKUS, "Obesity on children and adolescents: Understanding and treating the problem», Social Work in Health Care, 8 (1982): 11-29.

13 RockWell, «Big is not beautiful», New Society, 37 (1976): 175-177. 
tal de las investigaciones es que el conocimiento acerca de la distribución demográfica de la gordura liderará la mejora de los métodos de prevención en el tratamiento por «exceso de peso» ${ }^{14}$. A menudo, los datos epidemiológicos informan en muchos de los artículos, proporcionando una fuente útil de información de los estudios críticos. Werner Cahnman revisa rigurosamente algunos de los estudios en los que destaca un artículo de 1968, donde él señala el desinterés de la sociología en el tópico de la obesidad (un desinterés que parece continuar sin disminuir), y recomienda que el tratamiento de la obesidad sería mejorado por considerarlo sociogénico bastante más que considerar causas psicogénicas. Cahnman asume que debería considerarse cuestionable lo siguiente: el sobrepeso es la causa inmediata de obesidad; que una elevada incidencia de las enfermedades coronarias entre las clases socioeconómicamente bajas es atribuida al alto índice de obesidad entre esas clases; que la gente de clase baja está gorda porque tienen miedo a pasar hambre o porque se inclinan a favorecer la gratificación o ambos. Al mismo tiempo, Cahnman se cuestiona la noción de "peso normal» y discute algunas nociones cotidianas acerca de las consecuencias de sobrepeso para la salud. El define la naturaleza de la estigmatización del gordo/a, y sugiere que la reducción del estigma es la llave para la reducción de peso. Finalmente, hace un ruego a la tolerancia cuando no se pierde peso: "como en otras áreas problemáticas, así como en el área de la forma corporal y la apariencia - que es necesaria-, es un acuerdo de respeto mutuo común para la humanidad y para cada una/o de nosotras/os» ${ }^{15}$.

Albert Stunkard, psiquiatra de la Universidad de Medicina de Pennsylvania, ha escrito acerca de los aspectos sociales de la obesidad y de las dietas por décadas, aparentemente en ayuda de encontrar el tratamiento efectivo para la pérdida de peso $^{16}$. Stunkard, junto con Jeffrey Sobal, revisaron treinta estudios americanos, asociando la obesidad y el estatus socioeconómico, con datos en mujeres que revelan una consistente relación inversa entre clase social y obesidad. En algunos estudios —en otros países que han desarrollado el tema-, dos terceras partes de ellos, se desprende el mismo resultado. Estudios con datos de varones, a menudo, son inconsistentes, con alguna demostración de asociación positiva entre clase social y obesidad; otros demuestran una relación inversa y todavía algunos demuestran que no tienen relación. Curiosamente, mientras Sobal y Stunkard se reafirman en explicar la diversidad de los hallazgos para varones, éstos no parece que hagan de ello todo lo sospechoso acerca

14 Garn, Lavelle y Pilkington, "Obesity and living together», en Obesity and the family, editado por Kallen y Sussman, un número especial de Marriage and Family Review, 7 (1984); MCGEE y HALE, "Social factors and obesity among black women», Free Inquiry in Creative Sociology, 8 (1980): 83-87; SoBAL, "Group dieting, the stigma of obesity, and overweight adolescents", en Obesity and the family (Kallen y Sussman editores), un número especial de Marriage and Family Review, 7 (1984).

15 CAHnMan, «The stigma of obesity», Sociological Quarterly, 9 (1968): 283-299.

16 Stunkard está, a menudo, haciendo una crítica de los métodos de hacer dieta en la práctica médica. STUNKARD, The pain of obesity, Bulletin Oublishing, 1976. 
de los hallazgos en mujeres. En muchos, del ínfimo número de estudios de países desarrollados, encuentran asociaciones directas entre clase social y obesidad para ambos, mujeres y varones. Sobal y Stunkard explican el propósito del significado de las relaciones entre clase social y obesidad, en el proceso de revisión de algunos estudios del grado de estigmatización de la gordura en los Estados Unidos, y un número significativo de estudios psicológicos de adolescentes «su autoimagen con respecto al peso corporal». Ellos proponen cuatro posibles medidas/variables, ayudando a explicar la gran tendencia de las mujeres pobres en países desarrollados de ser gordas: dieta limitada, actividad física, movilidad social y herencia. Revisando los estudios sobre dietas, ellos concluyen que es significativa la alta incidencia de la clase social en las mujeres e inciden en que es mucho más probable que hagan dieta las mujeres de clase social media-alta que las de clase media-baja ${ }^{17}$.

Se han realizado, además, estudios de casos durante varios años en los Estados Unidos. En 1942, sociólogos como Useem y Tangent encuentran informes que inciden en el estudio de la estratificación social en la ciudad de Dakota del Sur. Más de la mitad muestra que las mujeres de clase social alta hacen dieta, mientras que ninguna de la muestra de mujeres de estatus bajo hace $\operatorname{dieta}^{18}$.

Sobal y Stunkard informan que la clase social está asociada (para ambos, mujeres y varones) con el crecimiento de la actividad física recreativa (aunque los niveles totales de actividad física por clase social no están reflejados). Además, unos pocos estudios ostentan ascendentemente el móvil de que la mujer es mucho más delgada que estable, por clase social o movilidad descendente. Ellos no consideran esto, sin darle importancia a la clase social. Las mujeres son gordas porque son pobres o pobres porque son gordas. Algunas investigaciones han encontrado la gordura, como influencia negativa en cuanto a las oportunidades de recibir educación y el acceso al empleo; y una evaluación social negativa de la gordura, en mujeres con movilidad ascendente a través de la probabilidad menor de casarse. Como Cahnman ha sugerido, "que la obesidad concierne sólo a las mujeres, no es más que un síntoma de ser de clase social baja, como una condena a ello» ${ }^{19}$. Además, las lagunas de este artículo conciernen a los estudios de exploración de la incidencia de la gordura entre varios grupos socioeconómicos. Esto no es culpa de Sobal y Stunkard, además, porque muchos de los estudios no han sido llevados a cabo. De este modo, nosotros sabemos acerca de la distribución de la gordura en la población como algo socialmente inexplicable, porque no tenemos la información acerca de la variación en el promedio de la existencia de personas gordas.

17 Sobal et al. (1984).

18 Ruth Useem, John Useem y Pierre TANGent, «Stratification in a prairie town», American Sociological Review, 7 (1942): 331-342.

19 Cahnman et al. (1968). 


\section{Estudios de estigmatización}

Una parte significativa de los estudios sociológicos han considerado ampliamente la estigmatización de la gordura. Una serie de estudios por Stephen Richardson ${ }^{20}$ cuestionaron a niños sobre las fotos de otros niños, en base a si ellos podrían elegir amigos a través de la visualización de dichas fotos. Estas representaban a seis niños con visibles minusvalías, incluyendo una pérdida de miembro o desfiguración facial. Las fotos de niños gordos eran consideradas las últimas como posibles amigos o compañeros de juegos. En el primer estudio, los niños entrevistados eran de 10 y 11 años, blancos y negros, de Puerto Rico, de varias clases urbanas y suburbanas. Similares estudios fueron realizados con niños de otras edades, clases y etnias y con adultos. Los resultados fueron más o menos similares en los últimos estudios, aunque los niños judíos figuraban con alto índice de gordura que otros niños que no fueron estudiados; y la categorización por variables de grupos de edad en otras investigaciones demostró que las chicas jóvenes de 12 años han pasado por la gordura, mientras que los chicos tienen menor tendencia a la obesidad. En ninguno de estos estudios, los chicos gordos están considerados como una preferencia para ser compañeros de juegos. Algunos de los trabajos son muy conocidos e informados por los investigadores interesados en estos supuestos; treinta años después del primer estudio que fuera publicado, está todavía vigente por los científicos sociales que escriben acerca del estigma ${ }^{21}$.

Los investigadores e investigadoras interesados en la estigmatización de las personas gordas han establecido un número de experimentos en los cuales varios sujetos fueron cuestionados por las características evaluativas de personas provistas de fotos, vídeos, dibujos e historias. Evaluaciones de gordos y «normales» fueron entonces comparadas, y las personas gordas recibieron una valoración menor. Recientes ejemplos — desde las publicaciones sociológicasincluyen reportajes de chicas adolescentes de una colección de fotos de chicas gordas y colegialas que utilizaron la colección de vídeos de chicas gordas para trabajar en tareas ${ }^{22}$. Tiggemann y Rothblum preguntaron a las colegialas en América y Australia por sus preferencias entre la gente gorda comparados con normales o sujetos afines: los gordos eran considerados como acogedores y amistosos, pero menos felices, menos autoconfidentes, más autoindulgentes, menos autodisciplinados, perezosos y menos atractivos. Mientras que los estu-

${ }^{20}$ Richardson et al. (1961) y Goodman et al. (1963); RiCHARDSON, "Age and sex in values toward physical handicaps", Journal of Health and Social Behavior, 12 (1970): 207-214; RICHARDSON, "Children's values and friendships: a study of physical disability», Journal of Health and Social Behavior, 12 (1971): 253-258.

${ }^{21}$ Goodman et al. (1963) y Richardson et al. (1970); Social Science Citation Index (1990).

22 DENJONG, "The stigma of obesity. The consequences of naive assumptions concerning the causes of physical desviance», Journal of Health and Social Behavior, 1 (1980): 75-87. LARKING y PINES, «No fat persons need apply: Experimental studies of the overweight stereotype and hiring preference», Sociology of Work and Occupations, 3 (1979): 312-327. 
diantes americanos, y especialmente las mujeres, fueron mejor considerados acerca del peso y más conscientes del cuerpo que las estudiantes australianas. Los varones y las mujeres de ambas culturas son significativamente más negativos hacia la gente gorda que hacia la gente «normal $»^{23}$.

Benson envió currículas y fotos de chicas y chicos supuestamente interesados/as en hacer licenciaturas y carreras técnicas en salud pública. Preguntó a las chicas que le ayudaran a valorar su oportunidad de éxito en ese campo. El pronóstico de fotos de chicas gordas era considerablemente más pesimista que el de las chicas normales; además, un número menor de sujetos volvieron a observar las fotos y cuando se encontraban con una foto de chicas gordas era valorada con cariño. Este estudio era especialmente interesante porque estos sujetos no eran estudiantes habituales de escuela o adolescentes, y porque en estos estudios las consideraciones de estigma no eran observadas en un laboratorio $^{24}$.

Algunos investigadores se habían interesado en las actitudes de los profesionales, especialmente los dedicados al cuidado de la salud, los cuales tratan a obesos/as. Los estudios de las actitudes profesionales eran particularmente útiles, por el fuerte rol que ejercen las definiciones de los médicos en las nociones de la gordura en la América cultural. El mejor conocedor de esos estudios estaba conducido, desde hace dos décadas, por los sociólogos George Maddox y Verónica Liederman, los cuales preguntaron a un grupo de médicos la opinión entre ellos mismos acerca de la obesidad (un "componente médico»), el promedio de pacientes obesos, a una paciente obeso, en concreto, y a una serie de parejas «adjetivamente» opuestas. Teniendo en cuenta que los médicos, arrolladoramente cargados de pacientes obesos, con poca fuerza de voluntad, considerados peligrosos y torpes, opinaron en general más negativamente de los pacientes que de su propia valoración y la de otros médicos, pero más negativos sobre todo acerca de los pacientes obesos ${ }^{25}$.

Najman envió a investigadores para entrevistar a doctores en Michigan y Australia preguntándoles acerca de las características que a ellos les gustaban menos de sus pacientes. La obesidad tenía el quinto lugar en Michigan, el sexto en Australia, menor antipatía que otras características como: el abuso del alcohol; cólera, hostilidad y ofensa; falta de higiene, drogas o abuso de estupefacientes, y leves desórdenes mentales; pero más antipatía que la hipocondría; mayor que los desórdenes mentales; músculo esquelético o cuestión étnica, etc. Sólo los datos de Australia estaban sujetos a un análisis detallado, pero entre los médicos, algunos eran menos hostiles a la obesidad que otros, y los médicos especialistas en endocrinología y cirugía eran más hostiles que psiquiatras y

${ }^{23}$ Tiggeman y Rothblum, "Gender differences in social consequences of perceived overweight in the United States and Australia», Sex Roles, 18 (1989): 75-86.

${ }_{24}$ Benson, Severs, Tatgenhorst y LoddengaArd, "The social cost of obesity: a nonreactive field study", Social Behavior and Personality, 1 (1980): 91-96.

${ }_{25}$ Maddox y Liederman, "Overweight as a social disability with medical implications", Journal of Medical Education, 44 (1969): 214-220. 
médicos de medicina general. Esta investigación realizada por Najman y sus colaboradores surge como si hubiesen sido motivados por la cuestión de la posibilidad de realizar una medicina mejor y ayudar más a la pérdida de peso. Ellos distribuyeron cuestionarios previos a una conferencia sobre las causas y el tratamiento de la obesidad. Lo que sucedió fue que asistieron más especialistas en nutrición y en programas de reducción de peso que de otra índole. Las cuestiones consideradas respuestas fiables de las causas y pronósticos de la obesidad eran respuestas indicativas de un arrollador consenso, como, por ejemplo, que la obesidad está causada por la comida como compensación, los problemas emocionales, la autoindulgencia y que inciden sobre todos los problemas familiares. Las preguntas a valorar por Najman y sus colegas se las sugirieron los contactos con otros profesionales en cuidados de la salud, especialmente los dedicados al tratamiento de la obesidad ${ }^{26}$.

Todos estos estudios contribuyen al entendimiento de la estigmatización de las personas gordas. Un número mayoritario de ellos consideran actitudes muy diferenciadas hacia las mujeres gordas y varones gordos, y unos pocos distinguen entre las actitudes hacia varones y mujeres "normales», por norma general. Esto es extraño, puesto que, a menudo, los investigadores que estudian las diferencias culturales en las actitudes acerca de la gordura se cuestionan: ¿de qué manera se estigmatiza según la clase social, la etnia y la edad en Estados Unidos? Los estudios utilizan entrevistas en profundidad, observación participante y otros métodos que reflejan muchas de las cuestiones como válidas y contrastadas, antes que, en circunstancias normales, sin investigaciones previas, ni cuestionarios, ni tampoco entrevistas, encontrar a las personas gordas útiles y aceptadas, pero esto no aparece en esta literatura.

\section{Efectos del estigma en las personas gordas}

Los trabajos sociológicos más interesantes en torno a la gordura han surgido con Natalie Allon y Marcia Millman ${ }^{27}$. Allon estaba preocupada por la obesidad y las dietas en el contexto de la sociedad americana, dentro de la cual, la mujer gorda sufre estigmatización y una reacción estructural de hostilidad en el mundo. Muchos de sus trabajos son estudios etnográficos de mujeres gordas y grupos de dietas. Ellas analizan las dietas como una forma de religión ritual, y la gordura es pecado y una identidad latente «servicio social benefactor» que los grupos de ditas sostenidos por sus miembros, más allá del propósito estatal de la pérdida de peso. Allon fue una propagandista para la Asociación Nacional para el Avance en la Aceptación de la Gordura» (NAAFA) ${ }^{28}$. En su análisis

26 Najman, Klein y Munro, "Patient characteristics negatively stereotyped by doctors», Social Science and Medicine, 16 (1982): 1781-1789.

27 Allon, «The stigma of overweight in everyday life», Obesity in Perspective (1983): 83-102; Millman, Such a pretty face, New York: W. W. Norton \& Company, 1980.

${ }^{28}$ NAAFA: National Association to Advance Fat Acceptance (Asociación nacional para el avan- 
del estigma social del gordo/a, en el volumen Recientes avances en la investigación en obesidad, Allon muestra gran ambición en dejar por sentado ocho hipótesis para ser investigadas por nuevos investigadores ${ }^{29}$. Desafortunadamente, Allon era consciente de que hacerlo sólo unas pocas mermaría su agenda de investigaciones propuestas antes que su profesión, que fue cortada por un accidente de coche en 1980. Una revisión de sus publicaciones y trabajos no publicados se puede encontrar en una edición especial de Marriage and family review en el tema de "Obesidad de familia», el cual está dedicado a Allon ${ }^{30}$.

Marcia Millman escribió Such a pretty face, que es un estudio supersociológico de las personas gordas. En su introducción al libro, Millman apunta que ella buscaba preguntas a cuestiones como "¿qué es esto de gustarme la vida de las personas gordas en mi propia sociedad cuando la obesidad y la gente gorda está desapareciendo?». Utilizando la observación participante y las entrevistas, ella desarrolló una visión sobre la experiencia de los gordos, que ambas funciones estaban descritas brillantemente y con una crítica social. Su libro revela que el camino de la gente gorda está victimizado por instituciones que proponen la existencia de su beneficio, como programas de pérdida de peso y campos para la farándula y el esperpento de los gordos/as. Esto revela mucho del gran estigma de la experiencia de las mujeres gordas comparable con los hombres gordos. Finalmente, Millman trabajó esto en los exámenes de NAAFA, en la organización que se resiste a la estigmatización de la gordura ${ }^{31}$. El trabajo de Allon y Millman está informado por ambas perspectivas, la de liberación de la mujer y la liberación de los gordos. De hecho, estos dos movimientos políticos tienen fuertes influencias de la teoría social acerca de la gordura, además del pequeño trabajo que han desarrollado los sociólogos.

\section{La liberación de la mujer}

La opresión por la apariencia para la mujer era temprana en lo concerniente al movimiento de liberación de la mujer y la estigmatización de la gordura. Es una de las muchas salidas que vienen encubiertas en el escrutinio feminis$\mathrm{ta}^{32}$. Algunos de los más conocidos análisis feministas de la fobia a la gordura, a menudo, continúan tomando referencias del trabajo social. Por ejemplo, Susie Ohrbach, una psicóloga que escribió Fat is a feminist issue (1978), desarrolla

ce en la aceptación de la gordura), creada desde 1969, con sede central en Sacramento. Esta asociación lucha en contra de la discriminación por razones de peso y tiene sedes en todos los estados de Norteamérica con más de medio millón de socias/os.

29 ALLON, "Fat is a dirty word: fat as a sociological and social problem», Recent Advances in Obesity Research (1974): 244-247.

30 Sobal et al. (1984).

31 Millman et al. (1980).

32 CHAPKIS, Beauty secrets: Women and the politics of appearance, Boston: South end Press, 1986; MunTER, "Fat and the fantasy of perception», en Pleasure and danger, editado por Vance, Boston: Routledge \& Kegan Paul, 1984, pp. 1781-1789. 
una crítica que demanda la posición de la mujer en la sociedad reclamando, entonces, el posicionamiento y la opción de ser gordo/a. Ella constata que la gordura es patológica, que a menudo está reflejada por los subtítulos de los libros (The anti-diet guide to permanent weight loss). Ohrbach comenta que algunas mujeres reconocen que las razones sociales de la gordura son elegir otras vías de protesta y conseguir la delgadez. Bajo la crítica desde la postura de los/las liberacionistas gordos/as, Ohrbach modificó este aspecto en la contraportada de Fat is a feminist issue I (1979), cambió el subtítulo por «una guía de autoayuda para comedores compulsivos». Sin embargo, la cubierta de la edición preliminar hablaba de "gordura como protección, sexo, maternal, fortaleza, afirmación y amor... tú puedes cambiar de reacción... en vista de que la pérdida de peso es una cosa buena...». Ohrbach sitúa su trabajo en un análisis feminista de los desórdenes en la alimentación, de los cuales la gordura es claramente un síntoma o indicio; su trabajo posterior fue Hunger Strike: The Anoretic's Struggle as a Metaphor for Our Age (1986).

La idea que se tiene de la obesidad y la anorexia como inadaptaciones familiares de opresión social de la mujer es común en las teorías feministas. Kim Chernin, por ejemplo, realiza una aproximación a la crítica psicoanalíti$\mathrm{ca} /$ social en su libro The Obsession (1981). Ella reivindica que la carne de la mujer representa a ambos, mujer y hombre, como un tremendo poder y sexualidad maternal. La mujer, teniendo mucha carne adicional, representa una amenaza para sus madres, siendo limitado el rol reproductivo en lugar de ser liberada. Esto es un ejemplo que ella explica acerca de la estigmatización social de las mujeres gordas y las mujeres obsesionadas con la delgadez. Por otra parte, Chernin considera la gordura como patológica, y supone que cuando las mujeres entienden las razones sociales, por sus miedos y conflictos, ellas frenarán el ser gordas. Chernin está poniendo juntas anorexia y gordura, con posteriores trabajos, acortando los problemas de la anorexia ${ }^{33}$.

Susan Bordo también habla de los desórdenes en la alimentación desde una perspectiva feminista. Bordo dice que ella no desea explicar las causas de esos desórdenes, pero quería tratar de observar los cuerpos delgados de las imágenes fotográficas de anuncios, incluyendo los de control del peso. Ella argumenta que nosotros/as percibimos la gordura como una fuerza hostil dentro de los propios cuerpos, el enemigo que estalla dentro pesadamente, y como algo blando y falto de movilidad o incapacidad de controlar a su propio enemigo. De este modo, ella observa la totalidad del deseo anterior, de tener un cuerpo flaco, para la mujer y el ahora popular y de moda musculoso cuerpo disciplinado liso y tirante. Bordo reclama que nosotros/as entendamos esta falta de control como una reflexión del buen estado de nuestras almas. Dibuja en la explicación de Chernin la dinámica de género de los desórdenes en la alimentación.

33 CHernin, The obsession: Reflections on the tyranny of slenderness, New York: Harper \& Row, 1986. CHERnin, The hungry self: women, eating and identity, New York: Times Books, 1985. 
Ella argumenta que la mujer requiere ejercer el control sobre sus propios cuerpos, en arreglo a no ser madre. Bordo sugiere que la bulimia es la incorporación de lo que Robert Crawford llama «la doble obligación del capitalismo» que nosotros producimos y que estamos suponiendo que será la disciplina autorechazada, y, como consumidores supondremos también que será desinhibido por la autoindulgencia. Bajo su punto de vista, la anorexia es socialmente más aceptada porque se aproxima a la cultura del cuerpo ideal, el obeso induce rabia por su apariencia indiferente de los estándares sociales, y consigue simpatía sólo de las/los que padecen el sufrimiento, independientemente de si ellas/os son felices porque son gordas/os ${ }^{34}$.

En algún trabajo histórico sobre feminismo contemporáneo ha sido mínimamente recogida la estigmatización de la gordura. Joan Brumberg, en su libro Fasting Girls, explicita una historia de la anorexia nerviosa. Explorando este objeto de estudio, sin embargo, Brumberg considera la visión histórica de la cultura americana de la gordura. Ella argumenta que es una adición a la hambruna el modelo de anoréxica. Esto es, hace el comentario de que la enfermedad es psicológica, biomédica y con componentes culturales; y que hay otras adiciones, como que prevalece el aumento del comportamiento anoréxico con la publicidad, y con el ayuno alimenticio ${ }^{35}$.

Para eliminar la obsesión americana por el peso, es necesario el conocimiento de la realidad económica y social, roles de género, maternales, paternales, desarrollo de la niñez y la familia y un conocimiento de factores biomédicos que influyen en la ganancia y la pérdida del peso.

\section{ALGUNOS EJEMPLOS DEL ESTIGMA DE LA OBESIDAD}

\section{Historias de vida}

Berta era una mujer inmensa. Pesaba más de 200 kilos. A pesar de todo, la gente disfrutaba con su compañía y ella tenía una intensa vida social. Un viernes por la noche, Berta y varios de sus amigos se pararon en una hamburguesería para tomar algo. A Berta no le gustaban los restaurantes de comida rápida por una buena razón: los asientos no eran adecuados para su tamaño. Pero ella era una buena amiga y quería ser agradable, así que no puso objeción para la elección de ese establecimiento. Berta encajonó su cuerpo en el asiento y tomó un batido y una hamburguesa. Una multitud típica del viernes por la noche esperaba de pie por una mesa, así que Berta y sus amigos acabaron de comer y empezaron a dejar los asientos para que los otros pudieran cenar. Pero los peores temores de Berta se hicieron realidad. Estaba tan fuertemente embutida entre la mesa y la silla que se había encajado completamente. Berta empezó a

34 Bordo, «Reading the slender body», Body Politics (1990): 83-113.

35 Brumberg, Fasting Girls, New York: New American Library, 1989. 
luchar por salir del asiento, sin éxito; sus amigos tiraban de ella, la empujaban, la contorsionaban, pero sin resultado. Ella estaba atrapada. Pronto todos los ojos del restaurante se dirigieron a la pobre Berta y a su lucha. Los mirones empezaron a reírse de ella. Las risitas se convirtieron en carcajadas y el restaurante retumbó de risa y de observaciones desagradables: "Dios, qué gorda"; "¡Qué pasa, cariño! ¿Demasiadas hamburguesas?; "Mira esa ballena atrapada»; "¿Cómo puede alguien ponerse tan gordo?»... La lucha de Berta la puso frenética, empezó a sudar a mares. Cada movimiento se convirtió en un acto de desesperación por liberarse de esa situación tan indignante. Finalmente, ella soltó el asiento de los tornillos y se puso en pie en medio del restaurante encerrada en la silla como si fuera un barril. A la gente le encantó, y se retorcían de risa, que cada vez era más intensa en volumen y estridencia. Mientras, Berta estaba indefensamente encajonada, ocupando el centro del salón. Uno de los amigos de Berta corrió a su coche, agarró un martillo y una llave inglesa, volvió a entrar y empezó a golpear el asiento, lo hizo añicos que cayeron al suelo liberando a la mujer de su tortura. Berta andaba pesadamente y salió a empujones entre la multitud que se reía y la miraba de reojo, y corrió hacia su coche con lágrimas en los ojos y la vergüenza ardiéndole en la garganta. El amigo que la liberó puso los trozos de la silla y de la mesa encima del mostrador los empleados, ahora enfadados, le pedían que pagara el asiento roto, pero él y los otros amigos de Berta simplemente se marcharon del restaurante.

Después de ese incidente, Berta apenas salió de casa. Dos meses después murió de un ataque al corazón; tenía 31 años.

\section{CONCLUSIONES}

La apariencia física es mucho más que sólo una forma corporal. Normalmente tendemos a creer que el perverso es feo y que el bueno es hermoso. Así pues, hay un trasvase del significado de lo físico al significado de lo comportamental y los rasgos físicos acaban convirtiéndose para los demás y para uno mismo en rasgos de personalidad ${ }^{36}$.

Goffman indicó en su ensayo sobre «los estigmas» que la gente suele ser más tolerante con las «desviaciones físicas», pero absolutamente intransigente con las «desviaciones caractereológicas». Sin embargo, en el caso de la obesidad (es decir, una condición física) parece no cumplirse, pues parece ser que se convierte en un vicio caractereológico. De este modo, el obeso no sólo sufre por su condición de obeso en sí; todos estos mecanismos sociales acaban siendo interiorizados por el propio individuo, que acaba sintiéndose culpable y responsable de todos los defectos que se le atribuyen ${ }^{37}$.

Sin embargo, no todos los obesos están igualmente estigmatizados. El cuer-

36 PerpiÑÁ (1989), Trastornos alimentarios: el estado de la cuestión, Valencia: Promolibro.

37 Goffman et al. (1990). 
po ha sido tradicionalmente un «valor» mucho más importante para la mujer que para el hombre. Por ello, las mujeres obesas están más discriminadas socialmente y presionadas por hacer dieta que los varones obesos y son ellas las que llenan las clínicas de control de peso.

Se puede afirmar que muchos de los síntomas que caracterizan a los obesos, tales como ansiedad, depresión, pobre imagen y baja autoestima, pueden ser el resultado más que la causa de su condición obesa. Es cierto que aunque existan grupos de obesos que por causas concretas, como la adolescencia, o las máximas presiones por conseguir un cuerpo delgado, sufren más desórdenes psicológicos, lo cierto es que no se ha encontrado ningún "perfil anormal de personalidad obesa», es decir, no están más perturbados psicológicamente que los sujetos con un peso normal, y no sufren una mayor incidencia de desórdenes emocionales que los no obesos. En ningún caso se puede afirmar que los rasgos de personalidad causen obesidad. Donde sí tiene sentido hablar de una posible "perturbación» psicológica de la obesidad es en las consecuencias que la obesidad tiene para el individuo. La presión del ideal estético, los estereotipos culturales están perjudicando a posteriori a gentes que no pueden desenvolverse entre los demás llevando encima un "cuerpo no grato".

En la actualidad se consolidan cada vez más las dietas alimenticias, los excesos y defectos dietéticos, el culto al cuerpo, las prácticas gimnásticas, el fitness y lo light; se han arraigado en nuestra sociedad como los grafitis y los pins. Ya no es importante el ser feliz y encontrarse bien consigo mismo, sino la proyección que tienen los demás sobre ti.

\section{BIBLIOGRAFIA}

Abraham, S., y Llewellyn-Jones, D. (1994): Anorexia y Bulimia. Desórdenes alimentarios, Madrid: Alianza Editorial.

Alemany, M. (1993): Obesidad y Nutrición, Madrid: Alianza Editorial.

Allon, Natalie (1973): "The stigma of overweight in everyday life», en George Bray (ed.), Obesity in perspective, Washington, DC: U. S. Government Printing Office, pp. 83-102.

- (1974): «Fat is a dirty word: Fat as a sociological and social problem», en A. Howard (ed.), Recent advances in obesity research, Westport, CN: Technomic Publishing Co., pp. 244-247.

- (1975): «Latent social services in group dieting», Social Problems, 23: 59-69.

- (1981): «The stigma of everyweight in everyday life», en B. J. Wolman (ed.), Psychological Aspects in Obesity, New York: Van Nostrand Reinhold, pp. 130-174.

BAnner, Lois (1983): American beauty, Chicago: University of Chicago Press.

Benson, Peter L.; Severs, Drew; Tatgenhorst, John, y LoddengaArd, Nancy (1980): «The social costs of obesity: a non-reactive field study", Social Behavior and Personality, 1: 91-96.

Blumer, Herbert (1971): «Social problems as colective behavior», Social Problems, 18: 298-306.

BORDO, Susan (1990): «Reading the slender body», en Mary Jacobus, Evelyn Fox Keller y Sally Shuttleworth (eds.), Body/politics, New York: Routledge, pp. 83-112.

BRumberg, Herbert (1971): "Social problems as collective behavior», Social Problems, 18: 298306.

Cahnman, W. J. (1968): «The stigma of obesity», Sociological Quarterly, 9: 283-299.

CHAPKIS, Wendy (1986): Beauty secrets: women and the politics of appearance, Boston: South end Press. 
CHERnin, Kim (1981): The obsession: reflections on the tyranny of slenderness, New York: Harper and Row.

- (1985): The hungry self: women, eating and identity, New York: Times Books.

Conrad, Peter, y Schneider, Joseph W. (1980): Desviance and medicalization, St. Louis: Mosby.

DENJONG, Willian (1980): "The stigma of obesity: The consequences of naive assumptions concerning the causes of physical desviance", Journal of Health and Social Behavior, 1:75-87.

ERnSBERGER, Paul, y HASKEW, Paul (1982): «Rethinking obesity: an alternative view of its health implications», The Journal of Family Practice, 14: 745-750.

FamuYiwa, O. O. (1988): "Anorexia in two Nigerians», Acta Psychiatrica Escandinavica, 78: 550-554.

FICHer, M. M. (1990): Bulimia nervosa: Basic Research, Diagnosis and Therapy, New York: Wiley.

Garn, S. M.; Lavelle, M., y Pilkington, J. J. (1984): «Obesity and living together», en Obesity and the family, editado por David Kallen y Marvin B. Sussman, en un número especial de Marriage and Family Review, 7.

Goffman, E. (1963): Estigma, Argentina: Anagrama, 1980.

Goode, E. (1983): «The fat admirer», Deviant Behavior, 4, 2 (Jan-Mar): 175-202.

Goodman, N.; Dornbusch, S. M.; Richardson, S. A., y Hartof, A. H. (1963): «Variant reactions to physical disabilities", American Sociological Review, 18: 429-435.

GUSFIELD, Joseph (1981): The culture of public problems: drinking-driving and the symbolic order, Chicago: University of Chicago Press.

Hamilton, Nina, y Zimmerman, Ruth (1985): «The interaction of marital power and weight loss success", Journal of Social Service Research, 8: 51-64.

Kallen, David, y Doughty, A. (1984): «Relationship of weight, the self perception of weight and self esteem with courtship behavior», en Obesity and the family, editado por David Kallen y Marvin B. Sussman, en un número especial de Marriage and Family Review, 7.

LARKIN, Judith Candib, y PINES, Harvey (1979): «No fat persons need apply: Experimental studies of the overweight stereotype and hiring preference», Sociology of Work and Occupations, 3: 312-327.

Lee, S.; ChiU, H. F. K., y Chen, C. (1989): «Anorexia nervosa in Hong Kong: Why not more in Chinese?», British Journal of Psychiatry, 154: 683-688.

MAdDox, G. L., y Liederman, V. (1969): «Overweight as a social disability with medical implications», Journal of Medical Education, 44: 214-220.

MALlER-JorIS, F. (1992): Divina, Madrid: Alfaguara.

MCGeE, Marsha, y Hale, Harry, Jr. (1980): "Social factors and obesity among black women», Free Inquiry in Creative Sociology, 8: 83-87.

MAYer, V. (1983): "The fat illusion», en L. Schoenfielder y B. Wieser (eds.), Shadow on a Tightrope, Iowa City, IA: Aunt Lute Book Company.

Millman, Marcia (1990): Such a pretty face, New York: W. W. Norton \& Company.

Najman, J. M.; Klein, D., y Munro, C. (1982): «Patient characteristics negatively stereotyped by doctors», Social Science and Medicine, 16: 1781-1789.

NASSER, M. (1988): «Eating disorders: The cultural dimension", Social Psychiatry and Psychiatric Epidemiology, 23: 184-187.

OHrbach, Susie (1978): Fat is a feminist issue: the anti-diet guide to permanent weight loss, New York: Paddington Press.

- (1979): Fat is a feminist issue: a self-help guide for compulsive eaters, New York: Berkley Books.

- (1986): Hunger strike: the anorectic's struggle as a metaphor for our age, London: Faber.

PERPIÑÁ, C. (1989): Trastornos alimentarios: el estado de la cuestión, Valencia: Promolibro.

RichaRdson, S. A. (1970): "Age and sex differences in values toward physical handicaps», Journal of Health and Social Behavior, 11: 207-214.

- (1971): "Children's values and friendships: a study of physical disability», Journal of Health and Social Behavior, 12: 253-258. 
Richardson, S. A.; Goddman, N.; Hastorf, A. H., y Dornbush, S. M. (1961): "Cultural uniformity in reaction to physical disabilities», American Sociological Review, 26: 241-247.

RoCKWELL, Joan (1976): «Big is not beautiful», New Society, 37: 175-177.

Rothblum, E. D. (1992): "The stigma of women's: social and economic realities», Feminism and Psychology, vol. 2.

Saldaña, C., y Rossell, R. (1988): Obesidad, Barcelona: Martínez Roca.

Schoenfielder, Lisa, y Wieser, Barb (eds.) (1983): Shadow on a Tightrope, Iowa City, IA: Aunt Lute Book Co.

SchWartz, Hillel (1986): Never satisfied, New York: The Free Press.

SCHWARTZ, Miriam A. (1984): «Expansionary America Tightens its belt: Social scientific perspectives on obesity", en Obesity and the family, editado por David Kallen y Marvin B. Sussman, en un número especial de Marriage and Family Review, 7: 49-63.

SEID, Roberta Pollack (1989): Never too thin, New York: Prentice Hall Press.

SobAL, J. (1984): "Group dieting, the stigma of obesity, and overweight adolescents: contributions of Natalie Allon to the sociology of obesity", en Obesity and the family, editado por David Kallen y Marvin B. Sussman, en un número especial de Marriage and Family Review, 7: 9-20.

Social Sciences Citation Index (1991), Philadelphia: Institute for Scientific Information.

SPITZACK, Carole (1990): Confessing excess: women and the politics of body reduction, New York: Suny Press.

StARger, S. F., y Burke, J. P. (1982): "A reexamination of body Build stereotypes», Journal of Research in Personality, 16: 435-446.

STEVEnSON, Kay M. (1978): "Obesity treatment: research and application», Social Work in Health Care, 4: 165-178.

StunKard, Albert J. (1976): The pain of obesity, Palo Alto, CA: Bulletin Oublishing Co.

Tiggeman, M., y Rothblum, E. (1989): "Gender differences in social consequences of perceived overweight in the United States and Australia», Sex Roles, 18: 75-86.

Useem, John; Tangent, Pierre, y Useem, Ruth (1942): «Stratification in a prairie town», American Sociological Review, 7: 331-342.

VAllejo NÁjera (comp.) (1991): Guía práctica de Psicología, Madrid: Temas de Hoy.

Wolf, Naomi (1991): El mito de la belleza, Barcelona: Emecé Editores.

ZAKUS, Gloria E. (1982): "Obesity on children and adolescents: Understanding and treating the problem", Social Work in Health Care, 8: 11-29. 


\section{RESUMEN}

El culto del cuerpo se ha convertido en nuestros días en un fenómeno social de gran significación. La presentación del yo físico adquiere una especial relevancia en los nuevos estilos de vida. Diversos grupos sociales (médicos, psicólogos, sociólogos, modistos, publicistas, estilistas, esteticistas, periodistas...) centran su interés en la definición social del "cuerpo». El objetivo es contribuir a la creación de un modelo idel único y generalizable, legitimando determinadas prácticas sociales dirigidas hacia este fin. La definición de este modelo está en manos de un grupo de «diseñadores de cuerpos» que asumen el papel de especificar los límites de la normalidad corporal en nuestra sociedad. La expresión corporal se ha convertido en un lenguaje que, en último extremo, manifiesta el status y la posición social.

Por otra parte, en las últimas décadas se plantea la cuestión de conocer cómo las diferencias biológicas entre los individuos se transforman en factores de discriminación social. En este sentido, diversos problemas de salud actuales chocan con la definición de «cuerpo» ideal. La gordura* es un buen ejemplo para comprender cómo las modas sociales utilizan la categorización de enfermedad «etiquetando" lo "normal» en un momento dado como "patológico» en otro. La exclusión social de las personas obesas, el rechazo a la obesidad y la construcción social de su figura marginal correspondiente, adquieren una relevancia merecedora de un análisis sociológico más exhaustivo. Por este motivo, es necesario comprender cómo se han impuesto criterios sociales para estigmatizar a personas cuyo peso no es un riesgo para la salud ni un obstáculo para su vida social...

\section{ABSTRACT}

Body worship has become nowadays a social phenomenon of major interest. The introduction of the physical "I» takes a special relevance in a new life styles. Several social groups (doctors, psychologist, sociologists, fashion designers, publicists, stylist, beauty consultants, journalist...) focus their interest on a socal definition on the "body». The goal is contribute to the settlement of a unique and general model, by legalizing some social practices aimed to that end. The definition of this model depends on a "body designers" group that itemize the limits of corporal normality in our society. Corporal expression has become a language that, at a last resort, states social position and status.

On the other hand, on the last decades the question of getting to know how biological differences between human being change into social apartheid factors is getting under way. In such a way, several health problems are in front of the ideal «body» definition. Fatness is a good example to understand how social fashions use the category of illness to «label» what is «normal» under specific circumstances as "pathological» under other ones. Social exclusion to fat people, the repulse to fatness and the social construction of its marginal figure acquire a relevance worth a more thorough sociological exam. Because of this, it is necessary to understand how social criteria have prevailed to stigmatize people whose weight is neither o risk to health nor and obstacle to their social life...

* El Diccionario de la Real Academia de la Lengua Española define el término Gordura como «grasa, tejido adiposo que normalmente existe en proporciones muy variables entre los órganos y se deposita alrededor de vísceras importantes. También como abundancia de carnes y grasas en las personas y animales». El término Obeso lo define como "dícese de la persona que tiene gordura en demasía». Y el término Obesidad como "cualidad de obeso». Una vez leídas estas definiciones se constata que la categorización de gordo y obeso está considerada como símil, pero la gordura siempre ha sido considerada como un término cultural y la obesidad un término médico. 